\title{
Searching for "Neuromarkers" Characteristic for Pathologic Changes in Schizophrenia by Using the Scaling Indices of the Cerebral Bioelectric Activity
}

\author{
Oleg Yu. Mayorov ${ }^{1,2,4^{*}}$ and Vladimir N. Fenchenko ${ }^{1,2,3}$ \\ ${ }^{1}$ Kharkiv Medical Academy of Postgraduate Education of the Ministry of Health, Ukraine \\ ${ }^{2}$ Institute for Medical Informatics and Telemedicine, Kharkiv, Ukraine \\ ${ }^{3}$ B.Verkin Institute for Low Temperature Physics and Engineering of the National Academy of Sciences, Kharkiv, Ukraine \\ ${ }^{4}$ Research Institute for Children and Adolescents Health Protection of the National Academy of Medical Sciences,
}

Kharkiv, Ukraine

\begin{abstract}
Introduction: The study is devoted to the development of new analytical procedures that would be fit for further study of the cerebral bioelectric activity (EEG) and may become reliable tools for diagnosing schizophrenia by using EEG.
\end{abstract}

Objective of the study: The study was aimed at detecting new "neuromarkers" suitable for diagnosing schizophrenia by using EEG.

Materials and methods: The subjects of the study were healthy individuals and patients with schizophrenia, both groups having been examined while either in the state of the undisturbed wakefulness or under mental exertion (backward counting in memory). Fractal characteristics of EEG traces has been determined by applying the Multifractal Detrended Fluctuation Analysis (MDFA).

Results: The dimensionality values of the dominant monofractal (the localization of the multifractal spectral function maxima) in patients with schizophrenia while in the state of undisturbed wakefulness could be increased in comparison with the healthy subjects. Have introduced a "decision-making rule" that allows to rate the status of tested subjects with special reference to the risk factors (suspected signs of schizophrenia) by employing the sets of data obtained by applying the procedures of multifractal analysis towards the EEG traces.

A diagnostic method allowing the detection of EEG signals pathologically deviating from normal ranges of values based on the use of multifractal characteristics has been suggested.

\section{Keywords}

EEG; Multifractality; Multifractal detrended fluctuation analysis; Multifractal spectrum; Classification; Nearest $k$; Neighbor algorithm method

\section{Correspondence to:}

Oleg Yu. Mayorov

Institute for Medical Informatics and Telemedicine, Kharkiv, Ukraine.

E-mail: o.y.mayorov@gmail.com

\section{EJBI 2018; 14(1):67-74}

Received: November 20, 2017

Accepted: February 14, 2018

Published: February 21, 2018

\section{Introduction}

Despite that numerous $[1,2,3,4,5,6,7,8,9,10,11]$ attempts have been made to analyze the bioelectric activity in patients with schizophrenia, no reliable criteria for evaluating EEGs were developed $[1,2,3,4,5,6,7,8,9,10$, 11]. EEG traces display a variety of diverse abnormalities, the degree of which is not expressed clearly and which are not significantly correlated with the degree of psycho-pathologic syndromes $[10,11]$. Doubtless, this disorder is associated with functional disintegration of cortical mechanisms (" $a$ disconnection hypothesis") [6], and it could be the underlying cause for other mental afflictions similar to schizophrenia [6, $7,8,9,10,11]$. In other words, not merely separate systems or structures are getting impaired, but their cooperative integrated functionality itself, which results in the onset of schizophrenia. All of the above calls for the development of new analytical procedures that would be fit for further study of the cerebral bioelectric activity and may become reliable tools for diagnosing schizophrenia by using EEG traces. 
The processes that occur within the brain, find their reflection reference group - 35 male patients aged 21-23) and the group in EEG signals, while their structure is complex and multifractal. of patients with schizophrenia that received no treatment (the Such processes can be quantified by way of factorization into trial group - 28 male patients aged 21-26, whose diagnosis has domains with variable local scale characteristics and the peculiar been confirmed clinically). The subjects in both groups have spectra of scaling parameters. Processes of this kind have been been examined while in the states of undisturbed wakefulness named multifractal - as opposed to monofractal ones, whose or under mental exertion (backward counting in memory). scaling parameters remain invariant respective to the scale of the given domain.

EEG traces were obtained by applying the monopolar acquisition technique adjusted to the 24-channel

Basic concepts of the multifractality theory have been electroencephalograph "DX-systems" (Ukraine), (Certificate formulated by Mandelbrot B [12, 13] and were shaped into a No 008/17/MD, Validity: to 20.06.2022, Ukrainian rigorous theory by Parisi G and Frisch U [14], Benzi R, et al. [15]. Certification Agency (UCA') Accredited by NAAU 10310

A wide-spread application of the multifractal analysis method towards arrays of biomedical data began following the publications by P.S. Ivanov et al. [16, 17], who have provided an experimental evidence for the fact that multifractal spectra of the human cardiorhythm could be efficiently used for diagnosing a variety of disorders.

Time-related dynamics of scaling patterns can be studied by a variety of approaches. Classical analytical procedures are applicable only towards parameters of stationary processes, whereas biological processes are distinguished by the heterogeneity and are not stationary.

Among the most efficient tools fit for the task of processing time series produced by such processes are the Wavelet Transform Modulus Maxima - WTMM [18, 19] and the method of the Multifractal Detrended Fluctuation Analysis - MDFA [20, 21, 22, 23].

Many contemporary studies have so far been focused primarily on the comparative statistical evaluation of the fractal parameters quantified by using these two methods [20, 24].

The majority of authors [18, 19, 20, 21, 22, 23], however, were employing simulative models based on the use of extended signals. In such cases, the randomly sampled measured values approximated the computed data sufficiently well. Meanwhile, time series of data obtained in medical-biological experiments are much less extended. The tool of preference for such series is the MDFA, which is, in fact, a kind of a variance analysis procedure intended for analyzing one-dimensional stochastic fluctuations [22]. The latter procedure allows for assessing the effects of prolonged correlations in non-stationary time series and provides arrays of fractal parametric values with more statistical regularity than those obtained by applying the WTMM. The correct selection of the signal domain within which the pattern of the scale variation would allow for the wavelet transformation, on the other hand, is a difficult task. If the assessed interval is relatively short, then, the values obtained by applying the WTMM procedure could reflect false multifractal properties [24, 25].

\section{Materials and Methods}

The study involved the group of healthy subjects (the S0/IEC 17065)) equipped with the averaged reference electrode ("10-20 system" according to Goldman and Offner). This version of the EEG acquisition technique provides an opportunity for tracking the cerebral activity locally - in the zones beneath the electrodes.

\section{Results}

\subsection{Formulation of the Problem. Analytical Methodology}

A study of the fractal characteristics pertaining to the time series of data representing the bioelectrical activity of the human brain (EEG traces) was conducted by applying the MDFA procedure. Diagnosing patients with schizophrenia, particularly at the initial stages of the disorder is quite a difficult task.

According to our initial assumption, the regulatory neurophysiological mechanisms would, under the normal circumstances, produce the dynamic signals that were going to be multifractal by nature, i.e. the multifractality would be further regarded as the "intrinsic" property of normally functioning control entities. Hence, measuring either the degree of the depletion of the multifractality or the variability of its parameters could provide invaluable diagnostic information concerning the status of the brain and its responses towards external stimuli.

EEG signals registered by one or other lead are quite complex structurally and are the results of the superimposition of a variety of signals originating from diverse sources (cerebral structures), which could be performing specific functions not necessarily correlated at a particular given point in time.

In clinical practice, only several specific frequency domains within the EEG signal patterns happen to deserve the attention of an examining specialist $\left(\delta, \theta, \alpha_{1}, \alpha_{2}, \beta_{1}, \beta_{2}, \gamma\right)$. The activity within each of the domains reflects the status of the brain and is associated with certain cerebral systems and mechanisms.

The factorization of the EEG signal into the component rhythms by applying the frequency filters would not, however, take into account a very peculiar phenomenon, namely, the 
presence in EEG signals of incorporated fragments (subsets) that are scale-invariant.

Scale invariance presupposes the invariance of fundamental characteristics of specific fragments in a situation of the scale transition; that is to say, the structure of a fragment on one given time scale would remain similar to its structure on an alternative scale. Notably, the time scale shift is possible within certain limits only, while the structure of the resulting pattern is not identical to that of the original fragment; statistical characteristics of both, however, do coincide.

Thus, the fine structure of EEG signals appears to contain characteristic spectra of fractal dimensions; based on these, an attribute vector intended for identifying cerebral conditions (including the status at the initial stages of schizophrenia) could be put in place.

Temporal sequences of data obtained by registering bioelectric potentials of the cerebral cortex, which, in fact, are arrays of values of the EEG signals measured at singular leads at equal time intervals, have been conventionally named "incremental" series.

The use of an MDFA procedure version adapted for such series is very well documented; its application towards the EEG signals, nevertheless, calls for specific modifications due to the fact that such signals contain rhythms of variable frequencies, amplitudes and, what is essential, of variable functional significance. The latter circumstance should always be taken into account whenever any kind of meaningful interpretation of multifractality of measured EEG signals is being attempted.

One fundamental step that the application of the MDFA towards the incremental series does involve is the extraction of a fluctuation profile from the initial series by using computed mean values. Further, this profile is expanded into non-overlapping segments each with the length of $s$ (as the length of the series is not always divisible by $s$, the number of points contained in the last domain is less than $s$, therefore, it is recommended that the expansion procedure be repeated, but, this time, from the opposite end of the sequence). Next, a local polynomial trend is determined for each of the segments and variance $\sigma_{n}(s)$ is assessed, where $n=1,2, \ldots, N_{s}$ is the number of the segment. Then, a so-called "deformed" variance.

$$
\sigma(q, s)=\left\{\frac{1}{N_{s}} \sum_{n=1}^{N_{s}} \sigma_{n}(s)^{q}\right\}^{\frac{1}{q}}
$$

calculated by raising a variance value for each segment to a power $q$ with a subsequent group averaging for all segments (at

$$
\sigma(0, s)=e^{\frac{1}{2 N_{s}} \sum_{n=1}^{N_{s}} \ln \sigma_{n}(s)}
$$
$q=0$ the following equation should be used):

$\sigma(q, s)$ within the wide range of values $S$ could be presented as an exponential function $\sigma(q, s) \approx s^{h(q)}$

where $h(q)$ is the generalized Hurst exponent, or scaling exponent.

In time series correspondent to a monofractal set, variance $\sigma_{n}(s)$ is similar for all segments, while the Hurst exponent is segment-invariant, and the generalized Hurst exponent is not dependent on the deformation parameter $q$.

In multifractal series at positive $q$ the major contributing components of $\sigma_{n}(s)$ are segments with greater variance levels $\sigma_{n}(s)$, while at negative $q$ the dominant role belongs to segments with lesser variances $\sigma_{n}(s)$.

In other words, at negative values of $h(q)$ the Hurst exponent $h(q)$ reflects the properties of segments with lesser fluctuations, while at positive values - of those ones with greater fluctuations.

Further, the standard interpretation of the scaling characteristics of a series would require a transition from the Hurst exponent $h(q)$ to the mass exponent [15] $\tau(q)=q h(q)-1$, which, at $q<<0$ and $q>>0$ increases linearly in direct proportion to $q$, while in the proximity to $q=0$, it acquires non-linear properties which causes the impediment of growth of $\tau(q)$ in proportion to the growth of the parameter $q$. In monofractals this domain is altogether absent, while $\tau(q)$ grows linearly and proportionally to $q$.

Mass exponent allows the calculation of the spectral function values $f(\alpha)=\alpha q(\alpha)-\tau(q(\alpha)), \alpha=\tau^{\prime}(q)$, the latter being the primary characteristic of multifractals [15].

The application of the MDFA (incidentally, as much as of the WTMM) towards the empirical values involves interpretational difficulties - in the event if a singularity spectrum $f(\alpha)$ is discrete, as the target of evaluation is the envelope of the local maxima, which would include a set of false points. Nonetheless, the analysis of multifractal EEG signals, which are generated by complex entities, typically produces continuous multifractal spectra that could be approximated by asymmetric spectral function plots, whereas the respective time series are significantly correlated temporally.

The spectral function $f(\alpha)$ defines a set of monofractals with dimensions $\alpha$, which configure a given multifractal. The position of its maximum would determine the dimensionality of a particular monofractal, whose contribution to the statistical summation would be dominant. The latter parameter is one of the most important characteristics of the multifractality of a given time series.

Another significant variable is the width of the multifractal

If the analyzed series could be reduced to a self-similar set spectra, i.e. the length of the domain within which the with long-range correlations, then the "deformed" variance function is positive. The values of this function would 
increase in a direct proportion to the degree of expression of the multifractality of the series in question.

In all probability, whether in the state of undisturbed wakefulness or while under mental exertion, patients with schizophrenia could display such cerebral activity patterns that may not be normally present in healthy individuals. Therefore, it is not unlikely that dimensions of dominant monofractals in subjects with schizophrenia could be, on the average, greater than in healthy study participants. Predictably, the multifractal spectral widths in schizophrenia should be abnormally greater, the causative factor being the existence of accessory ("coupling fault") connections among the systems that generate EEG signals.

In healthy individuals, the greater complexity of cortical reactions could be anticipated in response to the challenge of a mental exertion; as a consequence, the distribution of spectral function maxima could become right-skewed - hence, the dimensionality of the dominant monofractal may increase.

It could be speculated that in schizophrenic patients, in whose brain the coherent connections between cerebral structures had already been altered, such dislocation of the spectral function maxima could be of a different fashion; as much modified could be the shift at the level of multifractal spectral widths.

Thus, it appears that there is a certain degree of correlation between the dimensional variability of dominant multifractals and the increase of multifractality properties of the series. Thus, the assumption is that the most influential variable might be the value of the dimensionality of the dominant monofractal as measured at the level of a particular lead.

It turns out, then, that dimension values pertaining to dominant monofractals either in the state of undisturbed wakefulness or under the mental exertion could be arranged into series of elements ("neuromarkers"), which may further be used for rating the status of patients who have been a priori hypothetically diagnosed with initial stages of the schizophrenic disorder.

\subsection{Evaluation of the Parameters Characterizing} the Multifractality of the EEG Signal in Healthy and Affected Subjects

The objective of the study was the comparative evaluation of parameters that are employed as measures of multifractality of EEG signals obtained from healthy and affected subjects alike while in the state of undisturbed wakefulness or under the mental exertion (backward counting in memory).

Figure 1 shows a plot representing the characteristic dependency of the generalized Hurst exponent upon the deformation parameter (obviously, we get a regular Hurst exponent) obtained for a healthy individual in the state of undisturbed wakefulness and in the state of the mental exertion by using frontal leads (F3, F4), while the Figure 2 demonstrates the spectral function curve obtained by using the same leads.

The mental exertion was shown to have resulted in a significant transformation of the generalized Hurst exponent values along with the respective variation of spectral function values (based on use of frontal leads).

Of note: the generalized Hurst exponent values were dependent upon the variations of the deformation parameter both quantitatively and qualitatively. Such dependence revealed itself not only in dislocated spectral function maxima, but also in increased spectral widths. In other words, the greater was the dimensionality of the dominant monofractal the higher was the multifractality level, which, apparently, was suggestive of the added complexity of reactions occurring in the respective areas of hemispheres. At the same time, the frontal leads placed around the right hemisphere (F4) did not register any significant increase of spectral widths, while the degree of the individual variance in all groups was unusually high. This phenomenon is surely explicable, as the left hemisphere is known to contain a so-called "logic perception area" involved in logic-based activities (reading, linguistic perception, mathematical operations (calculus)), while the structures of the right hemisphere are dedicated to the spacial perception, are responsible for the perception of the "general" information, and are possibly capable of functioning in a variety of alternative ways for various individuals.

Figures 3 and 4 show the dependence of the generalized Hurst exponent values upon the deformation parameter variations along with the spectral function values obtained from the a patient with schizophrenia while in the states of undisturbed wakefulness and mental exertion (data obtained by using frontal leads (F3, F4)).

In this case, the values of Hurst exponent were at the significantly higher level than in a healthy subject. This fact suggests that the EEG traces may contain persistent series, which means that signals are positively autocorrelated, i.e. that the buildup of a signal would, most likely, produce a cumulative effect in the future and vice versa.

All of the above implies that the brain of a patient who is affected by the disorder and who is in the state of rest is running the processes different from cerebral reactions of healthy subjects.

The mental exertion, on the other hand, affects the dimensionality of a dominant monofractal so much less significantly, that any tangible advancement in complexity of the relevant cortical functionality could hardly be imagined.

Of note: the variance of values pertaining to the functions 

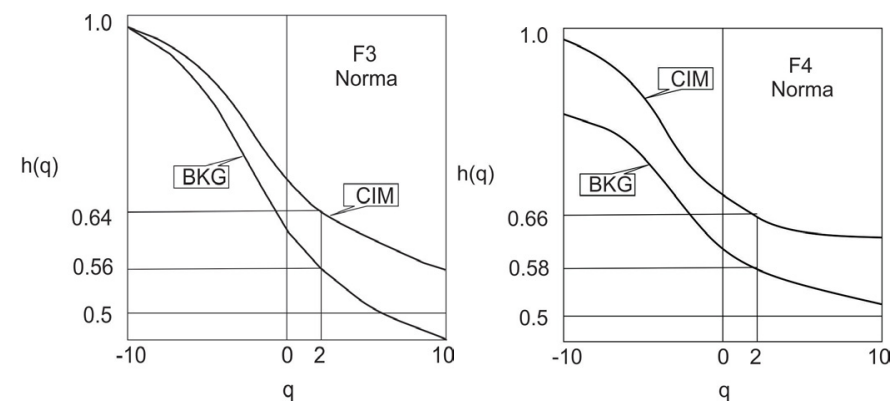

Figure 1: The comparison of generalized Hurst exponent values of a healthy subject in the states of undisturbed wakefulness (BKG) and mental exertion (CIM) (data obtained by using frontal leads (F3, F4)).

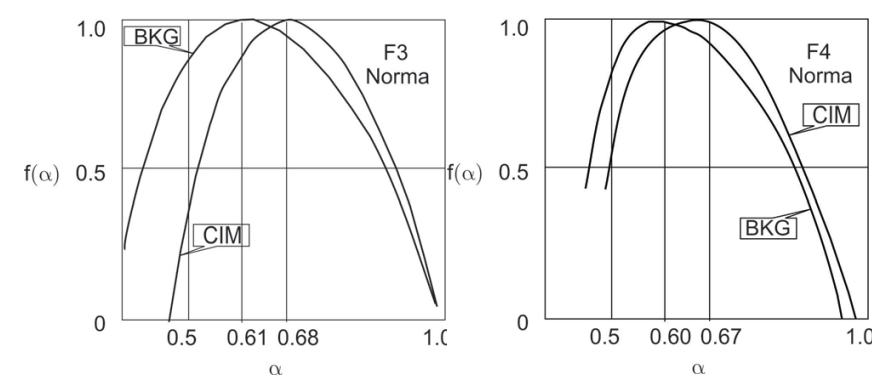

Figure 2: The comparison of spectral function values of a healthy subject in the states of undisturbed wakefulness (BKG) and mental exertion (CIM) (data obtained by using frontal leads (F3, F4)).

in question in patients with schizophrenia was significantly greater than in healthy individuals.

Tables 1 and 2 give calculated mean values of multifractality parameters of EEG signals measured in subjects while in the states of undisturbed wakefulness and mental exertion (the only data represented are sets measured by using the frontal (F3, F4) and parietal (P3, P4) leads registering the integrative activity and by using temporal leads (T3, T4) that provided correlates of emotional reactions).

In healthy subjects the transition towards the mental exertion has caused the dislocation of spectral function maxima, which was right-skewed at the level of all leads, i.e. the mental exertion has somewhat increased the dimensionality of the dominant monofractal.

In patients with schizophrenia the dislocation of the spectral function maximum under the mental exertion was inexplicit or, in some cases, even non-extant. In other words, the cortex of patients affected by the disorder was obviously devoid of the specific type of the connectivity between its structures.

In patients with schizophrenia, while in the state of undisturbed wakefulness, the dimensionality of the dominant monofractal (as a measure of the position of the spectral function maximum evaluated at all leads) appeared to have been greater than in healthy subjects (the level of significance being 0.95).

It should be emphasized, that in healthy individuals, even
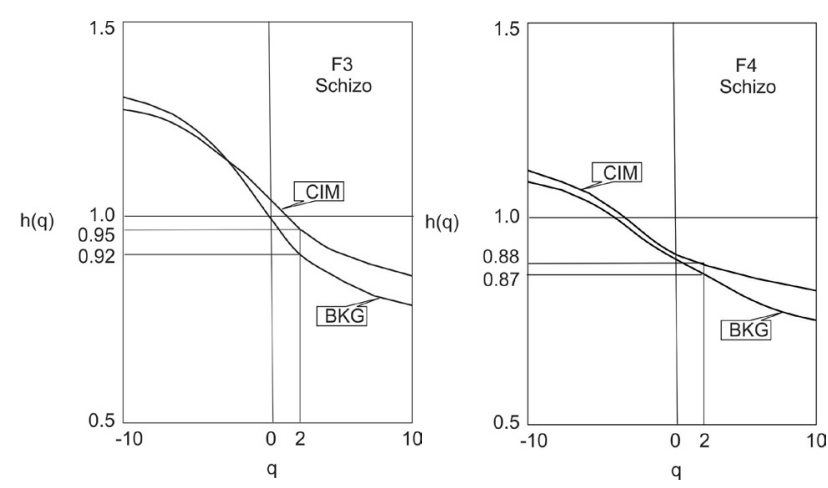

Figure 3: The generalized Hurst exponent values in a patient with schizophrenia in the states of undisturbed wakefulness (BKG) and mental exertion (CIM) (data obtained by using frontal leads $(\mathrm{F} 3, \mathrm{~F} 4))$.
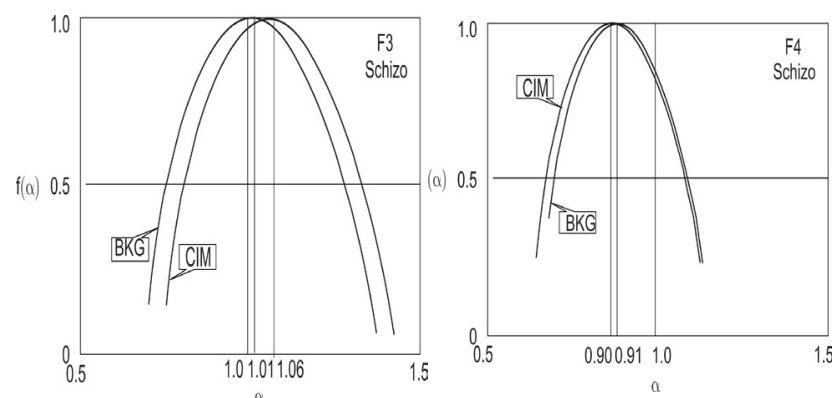

Figure 4: Spectral function values in patients with schizophrenia in the states of undisturbed wakefulness (BKG) and mental exertion (CIM) (data obtained by using frontal leads (F3, F4)).

while under mental exertion, the dimensionality of the dominant monofractal was at the lower level, than in patients with schizophrenia while they were at rest.

The multifractal spectral width values in healthy subjects, while they were switching to the mental exertion, have been somewhat growing, which was the evidence for the strengthening of connectivity between the signal-generating systems. In patients with schizophrenia subjected to the mental exertion, by contrast, the multifractal spectrum width values showed no tendency towards increase - much in accordance with our preliminary assumption. It should be noted that, while at rest, the group of patients with schizophrenia did show somewhat greater values of the multispectral width in comparison with the group of healthy subjects, which we explained by the presence of accessory ("coupling fault") connections between the signal-generating entities.

In conclusion, a neurophysiological interpretation of the results may be attempted. From the standpoint of a neurophysiologist, various local scale-sensitive properties of the EEG signals, the spectrum of their scale-related parameters, i.e. a so-called multifractal spectrum, reflect a series of rapidly interchanging mental processes. A multifractal EEG signal is an entity inclusive of several monofractal processes, which possess scaling characteristics remaining scale-invariant 
Table 1: Location of spectral function maximum.

\begin{tabular}{lllll} 
Leads & \multicolumn{2}{l}{ Mean value in healthy subjects } & \multicolumn{2}{l}{ Mean value in patients with the disorder } \\
& at rest (BKG) & under exertion (CIM) & at rest (BKG) & under exertion $($ CIM) \\
\hline F3 & $0.62 \pm 0.02$ & $0.69 \pm 0.02$ & $0.95 \pm 0.04$ & $0.97 \pm 0.09$ \\
F4 & $0.60 \pm 0.02$ & $0.67 \pm 0.04$ & $0.77 \pm 0.05$ & $0.81 \pm 0.08$ \\
T3 & $0.73 \pm 0.02$ & $0.77 \pm 0.02$ & $0.85 \pm 0.04$ & $0.82 \pm 0.06$ \\
T4 & $0.77 \pm 0.02$ & $0.82 \pm 0.04$ & $0.83 \pm 0.03$ & $0.82 \pm 0.07$ \\
P3 & $0.56 \pm 0.02$ & $0.59 \pm 0.01$ & $0.75 \pm 0.05$ & $0.76 \pm 0.07$ \\
P4 & $0.54 \pm 0.02$ & $0.58 \pm 0.01$ & $0.69 \pm 0.05$ & $0.69 \pm 0.06$ \\
Mean value for all leads combined & $0.64 \pm 0.04$ & $0.69 \pm 0.04$ & $0.79 \pm 0.05$ & $0.79 \pm 0.05$
\end{tabular}

Table 2: Values of the multifractal spectral width.

\begin{tabular}{|c|c|c|c|c|}
\hline \multirow{2}{*}{ Leads } & \multicolumn{2}{|c|}{ Mean value in healthy subjects } & \multicolumn{2}{|c|}{ Mean value in patients with the disorder } \\
\hline & at rest $(\mathrm{BKG})$ & under exertion (CIM) & at rest (BKG) & under exertion (CIM) \\
\hline F3 & $0.50 \pm 0.03$ & $0.50 \pm 0.05$ & $0.68 \pm 0.03$ & $0.70 \pm 0.05$ \\
\hline $\mathrm{F} 4$ & $0.47 \pm 0.03$ & $0.53 \pm 0.05$ & $0.54 \pm 0.04$ & $0.53 \pm 0.03$ \\
\hline T3 & $0.51 \pm 0.03$ & $0.53 \pm 0.03$ & $0.64 \pm 0.04$ & $0.58 \pm 0.05$ \\
\hline $\mathrm{T} 4$ & $0.46 \pm 0.02$ & $0.53 \pm 0.04$ & $0.55 \pm 0.03$ & $0.56 \pm 0.03$ \\
\hline P3 & $0.51 \pm 0.03$ & $0.51 \pm 0.03$ & $0.56 \pm 0.05$ & $0.52 \pm 0.03$ \\
\hline $\mathrm{P} 4$ & $0.51 \pm 0.04$ & $0.53 \pm 0.03$ & $0.63 \pm 0.05$ & $0.51 \pm 0.03$ \\
\hline $\begin{array}{l}\text { Mean value for all leads } \\
\text { combined }\end{array}$ & $0.49 \pm 0.10$ & $0.52 \pm 0.09$ & $0.60 \pm 0.05$ & $0.55 \pm 0.07$ \\
\hline
\end{tabular}

within any given range of scales. Amongst those there are isolated 3.3 Metric Classification of Healthy and dominant monofractals that are conditioned by prevailing mental Schizophrenic Patients by $k$-Nearest Neighbor reactions.

While in the state of undisturbed wakefulness, a significant difference between healthy test subjects and patients with schizophrenia has been detected - the dimensionality of scaling spectra pertaining to dominant monofractals in healthy individuals was lower than in patients with schizophrenia. This fact supports the assumption that the affected patients, while even in the state of undisturbed wakefulness, do experience effects of numerous mental reactions making them incapable of being relaxed even under favorable circumstances.

While under the mental exertion, the healthy subjects have displayed increased dimensionality of dominant monofractals, whereas the patients with schizophrenia showed no changes whatsoever; the greater dimensionality was detected either at rest or under mental exertion (backward counting in memory). These phenomena are apparently either the reflection of the stochastic nature of cerebral reactions (whether at rest or in the state of mental exertion, regardless) or the results of the impaired integrative functions. The lack of ability to relax or to get focused on the concrete task constitute the causative basis for the situation of the emotional distress.

\section{Algorithm Method}

Thereby, we arrive to the confirmation of the hypothesis, according to which the status of the cerebrum of an imaginary subject could be quite precisely described by using a twodimensional spatial set of characters, where a reference point is selected; the coordinates that define this point are the mean values of the dimensionality of the dominant monofractal for all leads combined either in the state of undisturbed awakening or under mental exertion. Figure 5 presents the set of such points obtained by studying select healthy subjects and patients with schizophrenia; the points have been plotted by using the following coordinates: "dimensionality values of the dominant monofractal measured at rest" against the "dimensionality values of the dominant monofractal under the mental exertion". It can be clearly seen that the points form the clusters in the vicinity of the mean values, thereby forming compact domains within the sample space. Notably, the clusters of points pertaining to the healthy subjects are quite dense; separation of any point from a cluster, therefore, could be interpreted as a deviation from the norm (by no means, though, should this separation be treated as a diagnostic sign of schizophrenia). Points pertaining to the 


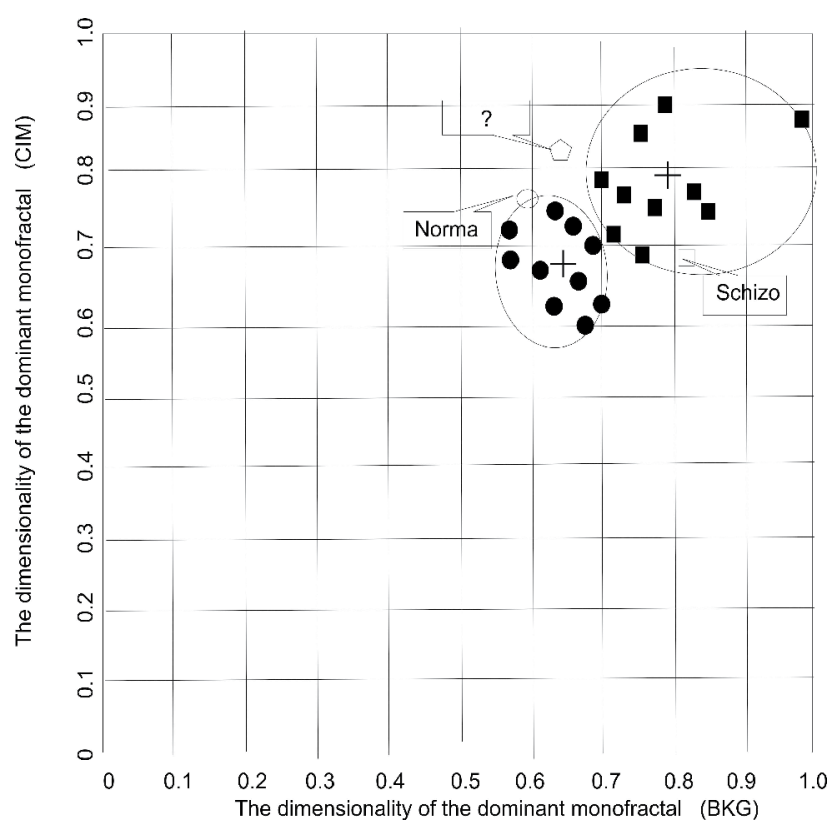

Figure 5: Filled characters correspond to patients belonging to reference groups (bullets - the state of norm, squares - schizophrenia), unfilled characters correspond to specific states of various groups of patients (bullets - the state of norm, squares - the state of schizophrenia is suspected, rhombuses - deviations from the state of norm are present).

patients affected by the disorder are somewhat "scattered" within the system of coordinates, and this variance is fairly natural. Migration of a point into this domain would be suggestive of the state of schizophrenia.

As the contours of domains correspondent to groups of healthy subjects and patients with schizophrenia alike are geometrically unsophisticated, the task of detecting suspected schizophrenia cases can be reduced to the application of metric classification procedures, e.g. of a relatively efficient and simple one $k$ - the nearest neighbor algorithm procedure ( $k$-nearest neighbor algorithm, KNN) [26]. This procedure is based upon the comparison of levels of probability with which a given tested individual may be theoretically associated with either a group of subjects with abnormalities unrelated to schizophrenia or with a group of those rated as suspected schizophrenia cases with special focus on the degree of affiliation between either of the groups and the majority of the test subject's nearest neighbors belonging to the "training set".

The distance to every "neighbor" is essential - the smaller the distance, the greater is the effect of the neighbor. Importantly, this algorithm is resilient against abnormal fluctuations and, if the necessity arises, can be easily adjusted in order to ensure the best possible precision of rating [27]. Schematically, the functionality of this algorithm is shown in Figure 5. Filled characters correspond to the patients from reference groups (bullets - healthy subjects, squares - patients with schizophrenia). Unfilled characters correspond to specific states in various groups of subjects (bullets
- the state is of norm, square - the state of schizophrenia is suspected, rhombuses - the patient's state is deviating from the norm, though schizophrenia is not suspected)

\section{Conclusion}

The results of the present studies have suggested that the dimensionality values of the dominant monofractal (the localization of the spectral function maxima) in patients with schizophrenia while in the state of undisturbed wakefulness could be increased in comparison with the healthy subjects. On the other hand, the transition towards the state of mental exertion (backward counting in memory) in healthy subjects has resulted in a more or less significant right-skewed dislocation of the spectral function maxima, while in patients with schizophrenia any significant redistribution was not observed.

Based on these observations, the authors have introduced a "decision-making rule" that allows to rate the status of tested subjects with special reference to the risk factors (suspected signs of schizophrenia) by employing the sets of data obtained by applying the procedures of multifractal analysis towards the EEG traces in the states of undisturbed wakefulness and mental exertion.

\section{Author Contribution}

Prof. OlegMayorovis the scientific supervisoroftheproject, selection and EEG examination of healthy and schizophrenics. Computer EEG analysis and interpretation of results. Mathematical support was given by Vladimir Fenchenko.

\section{Conflict of Interest}

The authors declare no conflicts of interest associated with this manuscript.

\section{References}

[1] Gandal MJ, Edgar JC, Klook K, Siegel SJ. Gamma synchrony: towards a translational biomarker for the treatment resistant symptoms of schizophrenia. Neuropharmacology. 2012; 62: 1504-1518.

[2] Castelnovo A, Ferrarelli F, D’Agostino A. Schizophrenia: from neurophysiological abnormalities to clinical symptoms. Front Psychol. 2015; 6: 478.

[3] Ferrarelli F. Sleep in Patients with Schizophrenia. Curr Sleep Medicine Rep. 2015; 1: 150-156.

[4] Ferrarelli F, Riedner B, Peterson MJ, Tononi G. Altered prefrontal activity and connectivity predict different cognitive deficits in schizophrenia. Human Brain Mapping. 2015; 36: 4539-4552.

[5] Kaskie RE, Graziano B, Ferrarelli F. Schizophrenia and sleep disorders: links, risks, and management challenges. Nat Sci Sleep. 2017; 9: 227-239. 
[6] Friston KJ. Theoretical neurobiology and schizophrenia. [18] Muzy JF, Bacry E, Arneodo A. Multifractal formalism Brain Med Bull. 1996; 52: 644-655.

[7] Woodruff P, Murray R. The aetiology of brain abnormalities in schizophrenia. In: Ancil RJ, Holliday S, Higenbottam J, editors. Schizophrenia: Exploring the Spectrum of Psychosis. Chichester, UK: Wiley; 1994, pp. 95-144.

[8] Andreasen NC. A Unitary Model of Schizophrenia: Bleuler's "Fragmented Phrene" as Schizencephaly. Arch Gen Psychol. 1999; 56: 781-787.

[9] Peled A. Multiple constraint organization in the brain: A theory for schizophrenia. Brain Res Bull. 1999; 49: 245-250.

[10] Coutin-Churchman P, Anez Y, Uzcategui M, Alvarez L, Vergara F, Mendez L. Quantitative spectral analysis of EEG in psychiatry revisited: drawing signs out of numbers in a clinical setting. Clin Neurophysiol. 2003; 114: 2294-2306.

[11] Gruzelier JH. Theory, methods and new directions in the psychophysiology of the schizophrenic process and schizotypy. Int J Psychophysiol. 2003; 48: 221-245.

[12] Mandelbrot B. Possible refinement of the lognormal hypothesis concerning the distribution of energy dissipation in intermittent turbulence. Statistical Models and Turbulence. 1972; 12: 333-351.

[13] Mandelbrot BB. Intermittent turbulence in self-similar cascades: divergence of high moments and dimension of the carrier. J Fluid Mech. 1974; 62: 331-358.

[14] Parisi G, Frisch U. On the singularity structure of fully developed turbulence. Proceedings of the International School of Physics “Enrico Fermi”. 1985; 84-87.

[15] Benzi R, Paladin G, Parisi G, Vulpiani A. On the multifractal nature of fully developed turbulence and chaotic systems. J Physics A: Mathematical and General. 1984; 17: 3521-3531.

[16] Ivanov PC, Nunes Amaral LA, Goldberger AL, Havlin S, Rosenblum MG, Struzik ZR et al. Multifractality in human heartbeat dynamics. Nature. 1999; 399: 461-465.

[17] Ivanov PC, Nunes Amaral LA, Goldberger AL, Havlin $\mathrm{S}$, Rosenblum MG, Stanley HE et al. From 1/f noise to multifractal cascades in heartbeat dynamics. Chaos. 2001; 11: 641-652.

[21] Kantelhardt JW, Koscielny-Bunde E, Rego HHA, Havlin $\mathrm{S}$, Bunde A. Detecting long-range correlations with detrended fluctuation analysis. Physica A. 2001; 295: 441-454.

[22] Peng CK, Havlin S, Stanley HE, Goldberger AL. Quantification of scaling exponents and crossover phenomena in nonstationary heartbeat time series. Choas. 1995; 5: 82-87.

[23] Stanley HE, Amaral LAN, Goldberger AL, Havlin S, Ivanov PCh, Peng CK. Statistical physics and physiology: Monofractal and multifractal approaches. Physica A. 1999; 270: 309-324.

[24] Oswiecimka P, Kwapin J, Drozdz S. Wavelet versus detrended fluctuation analysis of multifractal structures. Physical Review E: Statistical, Nonlinear, and Soft Matter Physics. 2006; 74: 161-203.

[25] Veneziano D, Moglen GE, Bras RL. Multifractal analysis: pitfalls of standard procedures and alternatives. Phys Rev E. 1995; 52: 1387-1398.

[26] Hastie T, Tibshirani R, Friedman J. The Elements of Statistical Learning. 2nd ed. USA: Springer; 2009.

[27] Berry Michael JA, Gordon Linoff. Data mining techniques: for marketing, sales, and customer relationship management. 2nd ed. USA: Wiley; 2004. 